PREHOSPITAL CARE

\title{
Effect of automatic external defibrillator audio prompts on cardiopulmonary resuscitation performance
}

\author{
L J Williamson, P D Larsen, Y C Tzeng, D C Galletly
}

See end of article for authors' affiliations

.....................

Correspondence to: Dr P Larsen, Section of Anaesthesia and Resuscitation, Wellington School of Medicine, PO Box 7343, Wellington, New Zealand; peter. larsen@wnmeds.ac.nz

Accepted for publication 30 July 2004

Objectives: To determine the effectiveness of the cardiopulmonary resuscitation (CPR) audio prompts in an automatic external defibrillator in 24 lay subjects, before and after CPR training.

Methods: Untrained subjects were asked to perform CPR on a manikin with and without the assistance of audio prompts. All subjects were then trained in CPR, and retested them eight weeks later.

Results: Untrained subjects who performed CPR first without audio prompts performed poorly, with only (mean (SD)) $24.5 \%$ (32\%) of compressions at the correct site and depth, a mean compression rate of 52 (31) per minute, and with $15 \%(32 \%)$ of ventilatory attempts adequate. Repeat performance by this group with audio prompts resulted in significant improvements in compression rate $(91$ (12), $p=0.0002$, paired $t$ test), and percentage of correct ventilations (47\% (40\%), $p=0.01$ paired $t$ test), but not in the percentage correct compressions $(23 \%(29 \%))$. Those who performed CPR first with audio prompts performed significantly better in compression rate (87 (19), $p=003$, unpaired $t$ test), and the percentage of correct ventilations (51 (34), $p=0.003$ unpaired $t$ test), but not in the percentage of correct compressions (18 (27)) than those without audio prompts. After training, CPR performance was significantly better than before training, but there was no difference in performance with or without audio prompts, although $73 \%$ of subjects commented that they felt more comfortable performing CPR with audio prompts.

Conclusions: For untrained subjects, the quality of CPR may be improved by using this device, while for trained subjects the willingness to perform CPR may be increased.

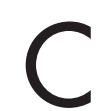
ardiopulmonary resuscitation (CPR), initiated by lay people, has been shown to significantly improve survival from out of hospital cardiac arrest, ${ }^{1}$ and the sooner CPR is started, the better the outcome. ${ }^{2}$ It is therefore important that members of the public are capable, and willing, to rapidly initiate effective CPR in response to collapse. In addition, out of hospital cardiac arrest survival is further improved with early defibrillation using automatic external defibrillators (AEDs). ${ }^{3}$

In the event that the initial defibrillatory shocks provided by an AED do not result in return of spontaneous circulation, or no shock is advised, the subsequent performance of CPR may be an important determinant of overall survival. With this in mind, some newer AED models, intended for lay people, have incorporated a CPR prompting system to guide rescuers in the performance of CPR. When used as part of manikin CPR training, an audio prompting system that guides the action sequences and the timing of chest compressions and ventilations, has been shown to speed the learning, increase retention of CPR skills, and improve CPR performance. $^{45}$

This study was conducted to find out if audio CPR prompts in the Laerdal Heartstart Plus AED improved CPR performance of untrained lay people, and of lay people who had received prior CPR training.

\section{METHOD}

Twenty four subjects with no previous experience of formal CPR training, were recruited for this study. Participants aged from 19 to 52 years, 18 women and six men. Each subject's knowledge of CPR was limited to media depictions.

Subjects were presented with a Laerdal Resusci-Anne manikin, and Laerdal Heartstart First Aid AED attached. They were informed that the manikin was unresponsive, not breathing, with no signs of life present. Each subject was then asked to perform CPR for 100 seconds, once without audio prompts and once with the assistance of audio prompts from the Laerdal Heartstart AED (table 1). The subjects were randomised such that half the subjects performed CPR first without audio prompts (group A) and half the subjects performed first with audio prompts (group B).

For each CPR attempt we recorded the number and rate of compressions, the proportion of compressions of correct depth and hand position, the number of ventilations, the proportion of ventilations of correct volume, and the compression: ventilation ratio, as measured and analysed by the Laerdal Skillmeter connected to the Resuci-Anne manikin.

Immediately after this initial assessment, all subjects were trained in the performance of CPR in accordance with the New Zealand Resuscitation Council's CPR guidelines for lay people. ${ }^{6}$ Training continued until subjects could perform CPR with the correct sequence, with greater than $60 \%$ of ventilations correct, correct compression site, rate of compressions between 90 and 110 , and greater than $60 \%$ of compressions correct.

Eight weeks after the first study day, 22 of the original group of 24 subjects returned to repeat the CPR assessments as on day one. On this occasion, subjects were randomised so that half of group A and half of group B performed first without audio prompts and remaining subjects performed first with audio prompts.

Statistical analysis was performed using StatView 5.0.

Abbreviations: AED, automatic external defibrillator; CPR, cardiopulmonary resuscitation 
Table 1 CPR prompts as given by Laerdal HeartStart First Aid AED

\begin{tabular}{ll}
\hline $\begin{array}{l}\text { Time } \\
\text { (seconds) }\end{array}$ & Instruction \\
\hline 0 & "Shock not advised" \\
2 & "Be sure the ambulance service has been called" \\
6 & "It is safe to touch the patient" \\
8 & "Check for signs of circulation. If no signs of circulation, \\
12 & start CPR" \\
15 & "For help with CPR press the flashing blue button" \\
24 & "Pinch nose. Tilt head. Lift chin and give two full breaths." \\
26 & "Breathe" \\
29 & "Place the heel of one hand in the centre of the chest, \\
& $\begin{array}{l}\text { between the nipples. Place your other hand on top of the } \\
\text { first. Push the chest down firmly } 5 \text { cm." }\end{array}$ \\
40 & "Keep in time with the beat." [15 audible beats then occur] \\
50 & "Pinch nose. Tilt head. Lift chin and give two full breaths." \\
59 & "Breathe" \\
61 & "Breathe" \\
63 & "Continue with compressions" [15 audible beats then \\
74 & occur] \\
83 & "Pinch nose. Tilt head. Lift chin and give two full breaths." \\
85 & "Breathe" \\
87 & "Breathe" \\
98 & "Continue with compressions" [15 audible beats then \\
Returns to shock advisory system
\end{tabular}

\section{RESULTS}

\section{Untrained lay rescuers}

\section{Group A: CPR without prompts}

Initial performance of CPR without prompts was poor (table 2). In the 100 seconds permitted, subjects performed (mean (SD)) 20 (16) compressions, with 24.5\% (32\%) of these correct. Hand position was correct in $54.1 \%$ (44.2\%) of compressions, and depth of compression correct for $36.0 \%$ (33.3\%) of compressions, with insufficient depth of compression $(60 \%(53 \%))$ more common than excessive compression $(4 \%(15 \%))$. The mean compression rate was 52 (31) per minute. Subjects attempted 4.3 (2) breaths, with 15\% (32\%) correct. Most subjects failed to open the airway correctly and little attention was paid to providing sufficient airflow to cause any noticeable manikin chest movement or the sound of airflow into the manikin. Subjects varied considerably in compression: ventilation ratio, with no one performing 15:2, and ratios ranging from 3:8 to 16:2.

Repeat performance of CPR with audio prompts resulted in significant improvements in the number of compressions performed (4l (7), $\mathrm{p}=0.0006$, paired $t$ test), compression rate (91 (12), p $=0.0002$, paired $t$ test), number of ventilations (6 (0), p $=0.01$, paired $t$ test), and the percentage of correct ventilations $(47 \%(40 \%), \mathrm{p}=0.01$ paired $t$ test $)$. There was no improvement in the hand position or total percentage of correct compressions (table 2). Eight of the 12 subjects performed CPR with a compression: ventilation rate ratio of $15: 2$, with one subject performing $14: 2$, one $13: 2$, and two subjects 10:2.

\section{Group B: CPR with AED audio prompts}

Subjects initial performance of CPR with prompts was significantly better than group A's performance without prompts in the number of compressions performed (39 (9), $\mathrm{p}=0.002$, unpaired $t$ test), compression rate (87 (19), $\mathrm{p}=0.003$, unpaired $t$ test), number of ventilations (6 (0), $\mathrm{p}=0.009$, unpaired $t$ test), and the percentage of correct ventilations (51 (34), $\mathrm{p}=0.003$ unpaired $t$ test). There was no improvement in the percentage correct hand position, the percentage correct compression depth, with insufficient depth of compression $(51 \%(46 \%))$ the most significant problem, or the total percentage of correct compressions (table 2). Six of the 12 subjects performed with a compression: ventilation rate ratio of 15:2, with the other six varying between $6: 2$ and 16:2.

Repeat performance of CPR by group B without prompts did not significantly differ from the initial performance with prompts (table 2).

When asked to comment on the audio prompts, all of the untrained subjects reported that these were very useful. However, four (17\%) participants expressed difficulty in being able to keep up with the speed at which the initial advice was given by the AED. Four subjects were unable to follow the AED metronome's beat in the way that it was intended.

Instead of using one beat per compression, giving a compression rate of 100 beats per minute, these subjects used one beat for compression and the next beat for relaxation, resulting in a compression rate of between 50 and 60 beats per minute. Three participants remarked on the depth to which the AED required them to compress; although each subject attempted to follow the device instructions, all mentioning that they believed a depth of $5 \mathrm{~cm}$ to be excessive, and queried the accuracy of the instruction.

\section{Trained lay rescuers}

At reassessment eight weeks after training there were no significant differences between the first and second attempts at CPR with audio prompts first, or no audio prompts first (table 2).

Both the initial attempts with and without audio prompts were significantly better than the corresponding attempts in the untrained group in terms of compression rate and the percentage of correct ventilations (table 2), but in neither case was there a greater percentage of correct compressions, with compressions of insufficient depth the most common error $(51 \%$ (37\%) for without audio prompts and 49\% (35\%) with audio prompts).

When asked to comment on the AED audio prompts, all subjects commented favourably, with most ( 16 of $22,73 \%$ ) stating that they felt more confident performing CPR with the audio prompts than without, and would be more likely to perform CPR if such a device were available.

\section{DISCUSSION}

Public access defibrillation programmes have reported that completely untrained subjects have used AEDs successfully. ${ }^{3}$ The addition of audio prompts to talk these untrained people through CPR may result in increased chance of survival for those subjects in whom defibrillation is not indicated or where the return of spontaneous circulation is not achieved in the first triplet of shocks.

We observed that in untrained subjects AED audio prompts significantly improved the performance of CPR. These improvements were seen in the total number and rate of chest compressions, and the number of correct ventilations performed. However, although the number of compressions improved, the proportion of compressions that were of sufficient depth was low, and unchanged.

For trained users there was no significant improvement in the measured quality of CPR with the addition of AED prompts. However, study participants expressed a very positive view of the CPR audio prompts, with most stating that they would be more willing, and confident, to perform CPR, if such a device was available. This may be important because fear of incorrectly performing CPR is a significant known barrier to bystander CPR. ${ }^{7}$

The aspects of CPR performance that remained poor, even with AED prompts, were adequacy of ventilation volume, and 


\begin{tabular}{|c|c|c|c|c|}
\hline \multirow[b]{2}{*}{ Attempt number } & \multicolumn{2}{|c|}{ Untrained rescuer } & \multicolumn{2}{|l|}{ Trained rescuer } \\
\hline & 1 & 2 & 1 & 2 \\
\hline \multicolumn{5}{|c|}{ \% Compressions correct depth: } \\
\hline Prompts first & $34.4(35.7)$ & $42.9(36.0)$ & $42.2(35.2)$ & $46.7(36.2)$ \\
\hline No prompts first & $36.0(33.3)$ & 30.7 (31.3) & $36.6(32.0)$ & $39.2(31.1)$ \\
\hline \multicolumn{5}{|c|}{ \% Compressions correct hand position: } \\
\hline Prompts first & $76.0(35.6)$ & $69.6(40.4)$ & $79.9(25.6)$ & $85.7(20.4)$ \\
\hline No prompts first & $54.1(44.2)$ & $67.8(42.7)$ & $81.1(27.9)$ & 71.7 (36.3) \\
\hline \multicolumn{5}{|c|}{$\begin{array}{l}\% \text { Correct compressions (position and } \\
\text { depth): }\end{array}$} \\
\hline Prompts first & $18.8(27.2)$ & $25.7(32.5)$ & $25.2(27.2)$ & $29.6(24.6)$ \\
\hline No prompts first & 24.5 (32.2) & $23.3(29.4)$ & $16.7(23.9)$ & $25.4(33.6)$ \\
\hline \multicolumn{5}{|c|}{ Mean compression rate: } \\
\hline Prompts first & $87.3(19.4)$ & $86.2(19.9)$ & $99.0(3.7) \ddagger$ & $99.1(7.1) \ddagger$ \\
\hline No prompts first & $52.3(31.4)+\dagger$ & $91.6(12.1)^{* * *}$ & $94.5(11.4) \pm$ & $93.1(15.2)$ \\
\hline \multicolumn{5}{|l|}{$\%$ Correct ventilations: } \\
\hline Prompts first & $51.3(34.4)$ & $41.6(33.3)$ & $84.8(22.9) \pm \ddagger$ & $77.2(31.0) \pm \ddagger$ \\
\hline No prompts first & $15.3(32.8) \mathrm{t \dagger}$ & $47.2(40.0)^{* *}$ & $55.5(43.3) \pm \ddagger$ & $65.8(36.5) \pm \ddagger$ \\
\hline
\end{tabular}

*Indicates significant difference $(p<0.05)$ between attempt 1 and attempt 2 within a group (either prompts first or no prompts first) with ${ }^{* *}$ indicating $p<0.01$ and ${ }^{* * *} p<0.001$, paired $t$ test. tindicates significant difference $(p<0.05)$ between groups (prompts first compared with no prompts first), with + tindicating $p<0.01$ and t†tp $<0.001$, unpaired $t$ test. $\neq$ Indicates significant difference $(p<0.05)$ between untrained and trained rescuers within a group (either prompts first or no prompts first) for a given attempt, with $\ddagger$ tindicates $p<0.01$, paired $t$ test.

compression depth. Volume is a difficult measure to convey as part of an audio prompting system, however the instruction "give two full breaths" does not convey a volume or an end point of the breath. A statement "blow until the chest starts to rise" may provide better a better descriptor of the intended volume of ventilation.

Although it might be expected that a $5 \mathrm{~cm}$ compression depth, as a simple linear measurement would be more straightforward than volume, compression depth was generally inadequate with or without prompts for both trained and untrained rescuers. This finding suggests that subjects may have difficulty translating a linear measurement into a vertical chest displacement. Furthermore, three participants questioned the depth to which they were advised to compress. Although each of these subjects attempted to compress to the advised depth they doubted the accuracy of the AED's instruction. This doubt may in some part be attributable to the participants' prior exposure to media depictions of cardiopulmonary resuscitation, where adequate chest displacement is unlikely to be depicted on actors.

Six of the 24 participants commented that there was insufficient time between the instructions for providing artificial ventilation, and the detailed description of chest compression performance. They felt they were unable to pay full attention to the compression instructions while completing the rescue ventilations, and this may have adversely affected subsequent chest compression performance.

For the prompts to significantly improve performance in regard to depth and volume, the prompts may need to offer better instruction on performance, and in addition to monitor performance and provide correctional feedback. Such a system in manikins was recently studied by Handley and Handley, ${ }^{8}$ who reported significant improvements in the ventilation volumes and chest compression depths in trained rescuers when using a system that gave correctional feedback. They suggested that such a system should be incorporated into AEDs.

An unexpected finding was the difficulty some subjects encountered with the timing system, specifically on whether to time each beat to a compression, or to use alternate beats for compression and relaxation. This led to compression rates of 50 or 100 beats per minute, depending on the chosen rhythm. Although most participants followed the instructions as they were intended and did not find any ambiguity in the instructions, the finding highlights the need for careful pre-market testing of statement interpretation to achieve optimal performance.

The Laerdal Heartstart AED audio prompt system used for this study took 98 seconds from completing the first set of shocks to returning to shock advisory mode, and reanalysing the patient's heart rhythm. This is significantly longer than the recommended one minute of CPR between shocks, ${ }^{9}$ and is significantly longer than the 75 seconds that elapses before a return to the shock advisory system using the Heartstart AED if the audio prompts are not activated. In addition, there is a considerable time delay from the delivery of shock to the start of CPR ( 15 seconds), during which time there would be no perfusion of the heart or brain.

In summary, the audio prompt system incorporated in the Laerdal Heartstart AED was highly rated by study participants, and resulted in significant improvements in some aspects of performance by untrained subjects. Performance of chest compression depth and ventilation volumes was not improved however, and there were significant delays in performance of CPR, and time to the second triplet of shocks. No significant improvement was noted in subjects who had been trained in CPR, although trained subjects indicated that they would feel more confident in performing CPR in a real situation with the assistance of audio prompts. We conclude that for untrained subjects, the quality of CPR may be improved by using this device, while for trained subjects confidence and willingness to perform bystander CPR may be increased. Both of these factors could contribute to increased survival from sudden cardiac arrest.

\section{Authors' affiliations}

L J Williamson, P D Larsen, Y C Tzeng, D C Galletly, Section of Anaesthesia and Resuscitation, Wellington School of Medicine, PO Box 7343, Wellington, New Zealand

Funding: This study was supported by a grant from the Wellington Surgical Research Trust and the Laerdal HeartStart First Aid Defibrillator was supplied by Laerdal, New Zealand.

Conflicts of interest: none declared. 


\section{REFERENCES}

1 Cummins RO, Eisenberg MS. Prehospital cardiopulmonary resuscitation. Is it effective? J Am Med Assoc 1985;253:2408-12.

2 Eisenberg MS, Bergner L, Hallstrom A. Cardiac resuscitation in the community. Importance of rapid provision and implications for program planning. J Am Med Assoc 1979;241:1905-7.

3 Caffrey SL, Willoughby PJ, Pepe PE, et al. Public use of automated external defibrillators. N Engl J Med 2002;347:1242-7.

4 Consensus statement. Guidelines 2000 for cardiopulmonary resuscitation and emergency cardiovascular care-an international consensus on science. Resuscitation 2000:46:29-71.

5 Wik L, Thowsen J, Steen PA. An automated voice advisory manikin system for training in basic life support without an instructor. A novel approach to CPR training. Resuscitation 2001;50:167-72.
New Zealand Resuscitation Council. Saving lives-New Zealand Resuscitation Council guidelines level 2. Wellington, New Zealand: New Zealand Resuscitation Council, 2000.

7 Shibata K, Taniguchi T, Yoshida M, et al. Obstacles to bystander cardiopulmonary resuscitation in Japan. Resuscitation 2000;44:187-93

8 Handley AJ, Handley SA. Improving CPR performance using an audible feedback system suitable for incorporation into an automated external defibrillator. Resuscitation 2003;57:57-62.

9 Monsieurs KG, Handley AJ, Bossaert LL. European Resuscitation Council guidelines 2000 for automated external defibrillation. A statement from the Basic Life Support and Automated External Defibrillation Working Group. Resuscitation 2001;48 207-9.

Committee on Publication Ethics Seminar 2005

Friday 11 March 2005, 9.30 am - 5 pm, BMA House, London

This year's seminar will focus on COPE's new Code of Conduct for Editors and interactive workshops on common ethical and editorial dilemmas. The seminar is for editors, authors, and all those interested in increasing the standard of publication ethics.

The Code aims to set a new basic standard for the ethical conduct of editors and sets out guidelines for quality and correcting the record, standing by decisions made, ethics committee approval, consent for publication confidentiality of submitted material, guidance to authors, pursuing misconduct, relationship to publishers, owners, and advertisers, and conflict of interest. The code also creates a mechanism to refer a complaint to COPE if an editor has breached the code.

The seminar will include:

- The new Code of Conduct for Editors

- Dr lona Heath, Chair BMJ Ethics Committee-research, audit, and ethics committee approval

- COPE's new website-full text and keyword searching for COPE's advice on specific issues, for example research misconduct, conflict of interest, and deception

- Interactive workshops-common ethical and editorial dilemmas for editors

- Opportunities to network with other editors and share your experiences and challenges

The seminar is free for COPE members and $£ 30.00$ for non-members. Numbers are limited and early booking is advisable. For registrations or more information please contact Sam Knottenbelt at cope@bmigroup.com or call 0207383 6602. For more information on COPE see www.publicationethics.org.uk/ 